\title{
Lattice Predictions for Hybrids and Glueballs.
}

\author{
Craig McNeile ${ }^{a}$
}

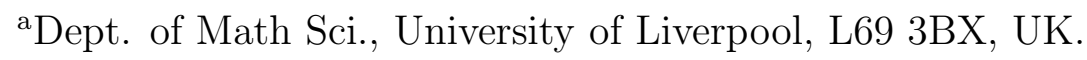

I review the results from lattice gauge theory for the properties of the light $1^{-+}$exotic state and $0^{++}$glueball.

\section{Introduction}

QCD is a simple elegant theory, but it is hard to solve it using anything other than perturbation theory. Quantities, such as masses, depend on the coupling ( $g$ ) like $M \sim$ $e^{-1 / g^{2}}$ [司], hence perturbation theory can't be used to compute the masses of hadrons such as the proton. Our inability to solve QCD non-perturbatively makes it hard to determine basic parameters of the quark sector of the standard model. For example, the allowed range on the strange quark mass in the particle data table [2] is 75 to $170 \mathrm{MeV}$; a range of almost $100 \%$.

A particularly good test of our understanding of the non-perturbative aspects of QCD is to study particles where the gauge field is excited somehow, and hence playing a more important dynamic role than in "standard" hadrons. Examples of such particles are glueballs (particles made out of the gauge fields) and hybrid mesons ( $\bar{q} q$ and excited glue). A very good overview of the interesting issues in hadronic physics, that contrasts the hadron spectroscopy approach to the study of confinement with the results from DIS type studies, is the white paper by Capstick et al. [3]. The only technique that offers any prospect of computing masses and matrix elements non-perturbatively, from first principles, is lattice QCD.

I review the results from the lattice for the lightest $0^{++}$glueball and $1^{-+}$exotic, as these states are the closest to being experimentally confirmed. Other recent reviews [4 6] of lattice results for hybrids and glueballs focus on different aspects of the subject.

\section{Lattice QCD calculations}

Many bound state properties of QCD can be determined from the path integral

$$
c(t) \sim \int d U \int d \psi \int d \bar{\psi} \sum_{\underline{x}} O(\underline{0}, 0) O(\underline{x}, t)^{\dagger} e^{-S_{F}-S_{G}}
$$

where $S_{F}$ is the fermion action (some lattice version of the continuum Dirac action) and $S_{G}$ is the pure gauge action. The path integral in eq. 1 is put on the computer using a clever finite difference formalism [1], due to Wilson, that maintains gauge invariance. The path integral in eq. 1 is evaluated using algorithms that are generalisations of the Monte Carlo methods used to compute low dimensional integrals. The physical picture for eq. [1 
is that a hadron is created at time 0 , from where it propagates to the time t, where it is destroyed. The physics from the calculation is extracted using a fit model [1]:

$$
c(t)=a_{0} \exp \left(-m_{0} t\right)+a_{1} \exp \left(-m_{1} t\right)+\cdots
$$

where $m_{0}\left(m_{1}\right)$ is the ground (first excited) state mass and the dots represent higher excitations. Although in principle excited state masses can be extracted from a multiple exponential fit, in practice this is a numerically non-trivial task, because of the noise in the data from the calculation. Any gauge invariant combination of quark fields and gauge links can be used as interpolating operators $(O(\underline{x}, t))$ in eq. 11 .

The fermion integration can be done exactly in eq. 1 to produce the fermion determinant. The determinant describes the dynamics of the sea quarks. In quenched QCD calculations, the determinant is set to a constant. Quenched calculations are roughly 1000 times cheaper computationally than the calculations that include the dynamics of the sea quarks. Quenched QCD gives quite a reasonable description of experiment. For example, the most accurate quenched calculation of the hadron spectrum, to date, has been completed by the CP-PACS collaboration [7]. From the masses of 11 light hadrons, they conclude that the quenched approximation disagrees with experiment by at most $11 \%$.

In an individual lattice calculation there are errors from the finite size of the lattice spacing and the finite lattice volume. State of the art lattice calculations in the quenched theory, run at a number of different lattice spacings and physical volumes and extrapolate the results to the continuum and infinite volume [7] limit. The increased computational costs of unquenched calculations means that most calculations are currently done at fixed lattice spacings. One of the most interesting unquenched calculations is being performed by the MILC collaboration [8]. MILC's calculations include $2+1$ flavours of sea quarks with a lattice spacing of $0.13 \mathrm{fm}$, box size of $2.6 \mathrm{fm}$, and the lightest ratio of the pseudoscalar to vector mass is 0.4 .

\section{Results for glueballs in quenched QCD}

Interpolating operators for glueballs are constructed for eq. 1 from closed loops of gauge links with the required $J^{P C}$ quantum numbers Some highlights of the results are that the lightest glueball is the $0^{++}$state with a mass of $1.64(4) \mathrm{GeV}$ [9]. The next lightest glueball is $2^{++}$. The ratio of the tensor to scalar glueball mass is $M_{2^{++}} / M_{0^{++}}=1.42(6)$ [9]. The spectrum of glueball states for other $J^{P C}$ quantum numbers with masses under $4 \mathrm{GeV}$ has been comprehensively mapped out by Morningstar and Peardon [10].

In the real world glueballs will decay to two mesons, hence they will have a decay width. Lattice QCD calculations are performed in Euclidean space, for convergence of the path integral in eq. 1. The Euclidean nature of lattice calculations makes the computation of inherently complex quantities such as decay widths more involved [11.

The GF11 lattice group computed the decay widths for the decay of the $0^{++}$glueball to two pseudoscalars [12 to be $108(28) \mathrm{MeV}$. Although the error bar is only statistical, it is encouraging that the width was small relative to the mass, so the $0^{++}$glueball may exist as a well defined state. The decay widths for individual meson pairs [13 did not agree with the predictions from the "flavour democratic" assumption. 
The experimental situation [2] for light $0^{++}$scalars is very interesting, because there are too many states to put into $\mathrm{SU}(3)$ nonets, as other particles with different $J^{P C}$ quantum numbers, such as the pseudoscalars, can be. The hadrons: $f_{0}(1370), f_{0}(1500)$, and $f_{0}(1710)$, have masses close to the mass of the quenched scalar $0^{++}$glueball.

In full QCD interpolating operators with $0^{++}$can be constructed from quarks and antequarks, such as $\bar{\psi} \psi$. In full QCD, the pure glue $0^{++}$operators will mix with the fermionic $0^{++}$operators. If the mixing is very strong, then the final $0^{++}$masses will have little to do with the quenched glueball masses.

Weingarten and Lee [14] studied the effect of mixing between the glueball and $\bar{\psi} \psi$ states in quenched QCD. They measured the correlation between the $0^{++}$glueball states and $\bar{\psi} \psi$ states in eq. 11. The results were expressed as a mixing matrix

$$
\left(\begin{array}{cc}
m_{g} & E(s) \\
E(s) & m_{\sigma}(s)
\end{array}\right)
$$

where $m_{g}$ is the glueball mass, $m_{\sigma}(s)$ is the mass of the non-singlet $0^{++}$state at strange, and $E(s)$ is the mixing energy. Weingarten and Lee measured: $m_{g}=1648(58) \mathrm{MeV}$, $m_{\sigma}(s)=1322(42) \mathrm{MeV}$, and $E(s)=61(58) \mathrm{MeV}$. The qualitative picture that emerges is that the $f_{0}(1710)$ is "mostly" $0^{++}$glueball, and the $f_{0}(1500)$ is "mostly" $\bar{s} s$. It is not clear whether $f_{0}(1500)$ being $\bar{s} s$ is consistent with its decay width 115.

The mixing energy $E(s)$ has large lattice spacing errors. For example at a lattice spacing of $a^{-1} \sim 1.2 \mathrm{GeV}$, the Weingarten and Lee [14] result is $E(s) \sim 0.36 \mathrm{GeV}$. This has been checked by another group's result [16] of $E(s) \sim 0.44 \mathrm{GeV}$.

The analysis of Weingarten and Lee [14] depends on the $0^{++}$states being well defined in quenched QCD. Eichten [17] et al. have shown that there is a problem with the nonsinglet $0^{++}$correlator in quenched QCD. The problem can be understood using quenched chiral perturbation theory. The non-singlet $0^{++}$propagator contains an intermediate state of $\eta^{\prime}-\pi$. The removal of fermion loops in quenched QCD has a big effect on the $\eta^{\prime}$ propagator. The result is that a ghost state contributes to the scalar correlator, that makes the expression in eq. 2 inappropriate to extract masses from the calculation. Eichten et al. [17] predict that the ghost state will make the $a_{0}$ mass increase as the quark mass is reduced below a certain point. This behaviour was observed by Weingarten and Lee 114 for small box sizes $(\mathrm{L} \leq 1.6 \mathrm{fm})$ for quark masses below strange. It is not clear how the problem with the non-singlet $0^{++}$correlator in the quenched approximation effects the results of Weingarten and Lee [14], however their most important results come from masses above the strange quark mass where the ghost diagram will make a smaller contribution that may be negligible

\section{Results for glueball masses in two flavour QCD}

The Weingarten and Lee [14] analysis predicted that the mixing of the $0^{++}$glueball and $\bar{\psi} \psi$ states is small. Parts of their calculation have been criticised in [16], however, the problems with the non-singlet $0^{++}$correlator [17] in the quenched QCD will make further progress in mixing in the quenched QCD difficult.

A lattice QCD calculation that included the dynamics of the sea quarks should just reproduce the physical spectrum of $0^{++}$states. Some insight into the composition of 
individual $0^{++}$states, such as whether a physical particle couples to $\bar{\psi} \psi$ or pure glue operators, could be studied by looking at the effect of decreasing the sea quark mass. For very heavy sea quark masses the theory is more like quenched QCD, where glueballs are distinct from $\bar{\psi} \psi$ operators.

Hart and Teper [18] found that the ratio of the $0^{++}$glueball mass in $n_{f}=2$ QCD to the quenched QCD result was: $M_{n_{f}=2}^{0^{++}} / M_{\text {quenched }}^{0^{++}}=0.84 \pm 0.03$ at a fixed lattice spacing of $0.1 \mathrm{fm}$. The $n_{f}=2$ results [18] for the mass of the $2^{++}$were consistent with the quenched value. As the lattice spacing dependence of the mass of the singlet $0^{++}$state in two flavour QCD and quenched QCD could be different, a definitive result will only come after a continuum extrapolation of the unquenched masses. In quenched QCD [9], the difference between the continuum extrapolated mass of $0^{++}$glueball mass and the mass at $0.1 \mathrm{fm}$ is of the order of $200 \mathrm{MeV}$. This is the same magnitude of the mass splittings between the masses of the experimentally observed particles $f_{0}(1500)$ and $f_{0}(1710)$. Although the current results for singlet $0^{++}$states are starting to be interesting, the lattice spacing used in unquenched calculations must be reduced before direct contact can be made to phenomenology.

The mass of the $0^{++}$glueball on the UKQCD data set are just degenerate with the mass of two pions [18. As the mass of the sea quarks is reduced, two pion states may effect the physics of singlet $0^{++}$states.

\section{Results for light $1^{-+}$exotic mesons}

The quark model predicts the charge conjugation $\left(\mathrm{C}=(-1)^{L+S}\right)$ and parity $(\mathrm{P}=$ $(-1)^{L+1}$ ) of a meson with spin $S$ and orbital angular momentum $L$. States with quantum numbers not predicted by the quark model, such as: $J_{\text {exotic }}^{P C}=1^{-+}, 0^{+-}, 2^{+-}, 0^{--}$are known as exotics [19]. Exotic states are allowed by QCD. Morningstar and Peardon [10] claim that there are no glueballs with exotic quantum numbers with masses less than 4 $\mathrm{GeV}$.

There are a number of different possibilities for the structure of an exotic state. An exotic signal could be: a hybrid meson, that is a quark and anti-quark with excited glue, or bound state of two quarks and two anti-quarks $(\overline{\psi \psi} \psi \psi)$.

One possible interpolating operator [20], that can be used in eq. 1, for a hybrid $1^{-+}$ particle is

$$
O_{1^{-+}}(\underline{x}, t)=\bar{\psi}(\underline{x}, t) \gamma_{j} F_{i j}(\underline{x}, t) \psi(\underline{x}, t)
$$

where $F$ is the QCD field strength tensor. If $F$ is removed from eq. 4 , the operator creates the $\rho$ particle. In this formalism a gauge invariant interpolating operator, for any possible exotic hybrid particle or four particle state can be constructed. The dynamics then determines whether the resulting state has a narrow decay width, hence it can be detected experimentally. In the large $N_{c}$ (number of colours) limit [19,21] both exotic hybrid mesons and non-exotic mesons have widths that are small compared to their masses.

There have not been many new calculations of the mass of the light $1^{-+}$hybrid recently. All the results from the various lattice QCD calculations, by UKQCD [22,23], MILC [20, 24] and SESAM [25] are essentially consistent with the mass of the $1^{-+}$state around $1.9(2) \mathrm{GeV}$ [4]. The interpolating operators used to create the exotic meson states in 
the MILC calculations 20] are different to those used in the UKQCD [22 and SESAM simulations [25], hence giving confidence that the systematic errors are under control. The results for the hybrid masses reported by Lacock and Schilling [25], include some effects from dynamical sea quarks. The recent results for the $1^{-+}$mass from calculations that used an asymmetric [26] lattice in time are consistent with the older results.

There are a number of experimental candidates for light $1^{-+}$states [2]. The E852 collaboration have reported [27] a signal for $1^{-+}$state around $1.6 \mathrm{GeV}$. There is also an experimental signal for a $1^{-+}$state at $1.4 \mathrm{GeV}$ [2].

There has been some recent work [28] on the quark mass dependence of the $1^{-+}$states. The lattice calculations are usually done at large quark masses and the results extrapolated to the physical quark masses. The conclusion of 28] was that the inclusion of the decay of the hybrid in the quark mass dependence of the exotic mass could reduce the final answer by $100 \mathrm{MeV}$. The predictions in 28] will be tested as the quark masses used in lattice calculations are reduced. The $1^{-+}$state at $1.4 \mathrm{GeV}$ seems low relative to the lattice results.

It is possible that the states seen experimentally are really $\bar{\psi} \psi \psi \psi$ states, in which case the operators used in the lattice simulations (eq. 1) might not couple strongly to them. Alford and Jaffe [29] studied $\overline{\psi \psi} \psi \psi$ operators with $J^{P C}=0^{++}$in a recent lattice calculation. The motivation was to gain insight into states such as the $f_{0}(980)$ that some people believe is not a $\bar{\psi} \psi$ meson, but a $\overline{\psi \psi} \psi \psi$ state. A similar lattice calculation could in principle be done for the $J^{P C}=1^{-+}$exotic.

To definitely identify a particle requires both the calculation of the mass as well as the decay widths. There has been very little work on strong decays on the lattice. The most obvious hadronic process to study using lattice gauge theory is the $\rho \rightarrow \pi \pi$ decay, however there have only been a few attempts to calculate the $g_{\rho \pi \pi}$ coupling [30,31. Michael discusses the problems with the formalism for hadronic decays on the lattice [11].

In the static quark limit the exotic states on the lattice are described by adiabatic potentials. The ground state of the static potential $\left(A_{1 g}\right)$ is the familiar Coulomb plus linear potential. The excited potential $\left(E_{u}\right)$ is a very flat potential, that can be used with Schrödinger's equation to predict the spectrum of heavy-heavy hybrids [4]. UKQCD [32] have investigated the de-excitation of the $E_{u}$ potential to the $A_{1 g}$ potential by the emission of a light quark loop. In the real world, the decays would correspond to $1^{-+} \rightarrow \chi_{b} \eta$ and $1^{-+} \rightarrow \chi_{b} S$ with $S$ a scalar and $\eta$ a pseudoscalar. The decay width of $1^{-+} \rightarrow \chi_{b} \eta$ and $1^{-+} \rightarrow \chi_{b} S$ transitions were less than $1 \mathrm{MeV}$ and around $80 \mathrm{MeV}$ respectively. The various approximations in the static limit mean that these widths have no direct relevance to experiment.

The MILC collaboration [20] have investigated the mixing between the operator in eq. [4 and the operator $\left(\pi \otimes a_{1}\right)$ eq. 5 .

$$
\bar{\psi}^{a} \gamma_{5} \psi^{a} \bar{\psi}^{b} \gamma_{5} \gamma_{i} \psi^{b}
$$

that has the quantum numbers $1^{-+}$. This type of correlator is part of the calculation required to compute the decay width of the $1^{-+}$state to $\rho$, and $a_{1}$. The more complicated part is to use eq. 5 in eq. 1 requires some clever numerical work. 


\section{Conclusions}

The glueball spectrum from quenched QCD is essentially complete. The key issues now are to quantify the mixing of the glueball states with $\bar{\psi} \psi$ operators and to determine decay widths.

\section{REFERENCES}

1. I. Montvay and G. Munster, Quantum fields on a lattice, Cambridge, UK: Univ. Pr. (1994) 491 p. (Cambridge monographs on mathematical physics).

2. Particle Data Group, D.E. Groom et al., Eur. Phys. J. C15 (2000) 1,

3. S. Capstick et al., Key issues in hadronic physics, 2000, hep-ph/0012238.

4. C. Michael, Glueballs, hybrid and exotic mesons, 2001, hep-ph/0101287.

5. C. Morningstar, 2001, nucl-th/0110074.

6. G.S. Bali, 2001, hep-ph/0110254.

7. K. Kanaya et al., Nucl. Phys. Proc. Suppl. 73 (1999) 189, hep-lat/9809146,

8. C.W. Bernard et al., Phys. Rev. D64 (2001) 054506, hep-lat/0104002,

9. M.J. Teper, Glueball masses and other physical properties of $\mathrm{su}(\mathrm{n})$ gauge theories in $\mathrm{d}=3+1$ : A review of lattice results for theorists, 1998, hep-th/9812187.

10. C.J. Morningstar and M.J. Peardon, Phys. Rev. D60 (1999) 034509, hep-lat/9901004,

11. C. Michael, Nucl. Phys. B327 (1989) 515.

12. J. Sexton, A. Vaccarino and D. Weingarten, Phys. Rev. Lett. 75 (1995) 4563, heplat/9510022.

13. L. Burakovsky and P.R. Page, Phys. Rev. D59 (1999) 014022, hep-ph/9807400,

14. W.J. Lee and D. Weingarten, Phys. Rev. D61 (2000) 014015, hep-lat/9910008,

15. F.E. Close and A. Kirk, Eur. Phys. J. C21 (2001) 531, hep-ph/0103173,

16. C. McNeile and C. Michael, Phys. Rev. D63 (2001) 114503, hep-lat/0010019,

17. W. Bardeen et al., Phys. Rev. D65 (2002) 014509, hep-lat/0106008,

18. A. Hart and M. Teper, Phys. Rev. D65 (2002) 034502, hep-lat/0108022,

19. T.H. Burnett and S.R. Sharpe, Ann. Rev. Nucl. Part. Sci. 40 (1990) 327.

20. MILC, C. Bernard et al., Phys. Rev. D56 (1997) 7039, hep-lat/9707008.

21. T.D. Cohen, Phys. Lett. B427 (1998) 348, hep-ph/9801316.

22. P. Lacock et al., Phys. Lett. B401 (1997) 308, hep-lat/9611011.

23. P. Lacock et al., Phys. Rev. D54 (1996) 6997, hep-lat/9605025.

24. C. McNeile et al., Nucl. Phys. Proc. Suppl. 73 (1999) 264, hep-lat/9809087,

25. P. Lacock and K. Schilling, Nucl. Phys. Proc. Suppl. 73 (1999) 261, hep-lat/9809022,

26. Z.H. Mei and X.Q. Luo, 2002, hep-lat/0206012.

27. G.S. Adams et al., Phys. Rev. Lett. 81 (1998) 5760,

28. A.W. Thomas and A.P. Szczepaniak, Phys. Lett. B526 (2002) 72, hep-ph/0106080,

29. M.G. Alford and R.L. Jaffe, Nucl. Phys. B578 (2000) 367, hep-lat/0001023,

30. S. Gottlieb et al., Phys. Lett. 134B (1984) 346,

31. R.D. Loft and T.A. DeGrand, Phys. Rev. D39 (1989) 2692,

32. C. McNeile, C. Michael and P. Pennanen, Phys. Rev. D65 (2002) 094505, heplat/0201006, 